\title{
躍iresalce
}

\section{Estudo comparativo de aplicativos móveis disponíveis para pacientes transplantados renais}

\author{
COMPARATIVE STUDY OF MOBILE APPS AVAILABLE FOR KIDNEY TRANSPLANT PATIENTS
}

\author{
Alan Rodrigues da Silva', Geraldo Bezerra da Silva Junior², Klébia Magalhães Pereira \\ Castello Branco ${ }^{3}$
}

\begin{abstract}
1 Farmacêutico. Departamento de Transplantes, Universidade Estadual do Ceará- UECE. ORCID: https://orcid.org/0000-0002-9633-363X

Email: alan_rodrigues.2010@yahoo.com.br 2 Doutor. Universidade de Fortaleza (UNIFOR). ORCID: https://orcid.org/0000-0002-8971-0994 Email: geraldobezerraj@@unifor.br

${ }^{3}$ Doutora. Hospital Dr. Carlos Alberto Studart Gomes. ORCID: https://orcid.org/0000-0002-6201-3283

Email: klebiacb@gmail.com
\end{abstract}

Correspondência: Universidade Estadual do Ceará Avenida. Dr. Silas Munguba, 1700 - Itaperi, Fortaleza CE, 60714-903.

Copyright: Esta obra está licenciada com uma Licença Creative Commons Atribuição-Não Comercial 4.0 Internacional.

Conflito de interesses: os autores declaram que não há conflito de interesses.

\section{Como citar este artigo}

Silva, AR da; Silva Junior, GB da; Branco, KMPC. Estudo comparativo de aplicativos móveis disponíveis para pacientes transplantados renais. Revista de Saúde Digital e Tecnologias Educacionais. [online], volume 5, n. 3. Editor responsável: Luiz Roberto de Oliveira. Fortaleza, dezembro de 2020, p. 01-15. Disponível em: http://periodicos.ufc.br/resdite/index. Acesso em "dia/mês/ano".

Data de recebimento do artigo: 08/06/2020

Data de aprovação do artigo: 11/09/2020

Data de publicação: 31/12/2020

\section{Resumo}

Objetivo: Avaliar os diferentes aplicativos disponíveis para pacientes que passaram por um transplante renal e propor melhorias a essas novas tecnologias. Métodos: Trata-se de uma pesquisa qualitativa, realizada a partir de uma revisão de aplicativos em smartphones usando a técnica benchmarking, pois esta é uma forma de estratégia que busca melhoria contínua, além de um importante elemento para aprimorar o mercado competitivo. Além disso, realizada avaliação de usabilidade e utilização da técnica SCAMPER. Resultados: Inicialmente, por meio da busca realizada com auxílio dos descritores, em inglês e português, foram identificados 943 aplicativos, sendo selecionados sete para o estudo. Quanto ao idioma, havia predomínio na língua inglesa $(86 \%)$. No teste de usabilidade apenas três $(42,9 \%)$ atingiram escore de corte de 68 pontos do System Usability Scale (SUS), dito como satisfatório. A técnica SCAMPER possibilitou a transparência entre as possíveis transformações e as modificações favoráveis e oportunas para as tecnologias móveis. Conclusão: Os resultados sugerem que é necessário desenvolver melhorias nos aplicativos já existentes, além de conceber ideias inovadoras para um protótipo destinado aos pacientes transplantados, resultando em ferramentas para melhor adesão farmacológica.

Palavras-chave: Aplicativos Móveis.

Benchmarking. Transplante de Rim. 


\section{Abstract}

Objective: To evaluate the different applications available to patients who have undergone a kidney transplant and propose improvements to these new technologies. Methods: This is qualitative research, carried out through a review of applications on smartphones using the benchmarking technique, as this is a form of strategy that seeks continuous improvement, in addition to being an essential element to improve the competitive market. In addition, usability and use of the SCAMPER technique was assessed. Results: Initially, through the search carried out with the help of descriptors, in English and Portuguese, 943 applications were identified, seven of which were selected for the study. As for the language, the English language was predominant (86\%). In the usability test, only three (42.9\%) achieved a cut-off score of 68 points of the System Usability Scale (SUS), said to be satisfactory. The SCAMPER technique enabled transparency between possible transformations and favorable and timely modifications for mobile technologies. Conclusion: The results suggest that it is necessary to develop improvements in existing applications, in addition to devising innovative ideas for a prototype for transplant patients, resulting in tools for better pharmacological adherence.

Keywords: Mobile Applications. Benchmarking. Kidney transplantation.

\section{Introdução}

O uso de tecnologias em saúde, destacando as mídias de informação, é bastante disseminado no âmbito da atenção ao paciente, proporcionando conteúdos padronizados, linguagem e design adequados ${ }^{1}$. Segundo Gálvan et al. ${ }^{2}$, o uso de tecnologias tem impacto na redução de riscos para o paciente, proporcionando ações preventivas por meio do acesso remoto entre os profissionais e os pacientes, tornando-se promissora por ofertar uma cobertura de serviços em saúde além de fornecer informações clínicas efetivas.

Frente aos avanços das tecnologias, as informações direcionadas aos pacientes na forma escrita, como folhetos educativos e panfletos, são pouco visibilizadas, tendo os aplicativos (APPs) de tecnologia móvel maior aceitação pelos pacientes, demonstrando maior efetividade nos resultados terapêuticos. Estas tecnologias possuem particularidades como acesso rápido e fácil, além de fazerem parte da rotina da maioria da população³.

A complexidade do tratamento imunossupressor, possíveis interações fármacofármaco e fármaco-alimento, reações adversas e contraindicações são informações presentes nos aplicativos. A tecnologia Mobile-Healthé uma nova estratégia que auxilia no uso correto e seguro da terapia e promove a adesão medicamentosa, além de contribuir no gerenciamento de doenças e no controle dos sinais vitais dos pacientes ${ }^{4,5}$

o benchmarking é uma técnica que avalia as propostas disponíveis no mercado, possibilitando a identificação de recursos que justifiquem o êxito ou insucesso de determinado produto ou serviço. Atua como um instrumento de busca, possibilitando realizar comparações com a finalidade de proporcionar melhorias e ideias inovadoras, uma vez que dá suporte na tomada de decisões em ambientes em constante transformações ${ }^{6}$. 
Diante dos novos recursos tecnológicos, o benchmarking surge como uma técnica de busca eficaz para correlacionar os principais aplicativos de interesse do pesquisador, trazendo resultados através de revisões que selecionam aplicativos por meio da avaliação comparativa e discussão particular de cada APP identificado. Mediante esse seguimento fornecem APPs com melhores evidências e funcionabilidades ${ }^{7}$.

Houve um crescimento significativo no uso de dispositivos móveis no Brasil e em todo o mundo, fazendo-se necessária a avaliação e a disponibilização de APPs para pacientes transplantados renais. Nesse escopo, o estudo objetivou avaliar os diferentes aplicativos disponíveis para pacientes transplantados renais e propor melhorias a essas novas tecnologias.

\section{Métodos}

Trata-se de uma pesquisa qualitativa, realizada a partir de uma revisão de aplicativos em smartphones dividida em três etapas básicas: identificação dos aplicativos usando o benchmarking, avaliação de usabilidade e a técnica SCAMPER. A primeira etapa consistiu em utilizar a técnica benchmarking, com a finalidade de identificar os aplicativos nas principais lojas virtuais e explorar os conceitos funcionais dos que estavam disponíveis ${ }^{7}$. 0 benchmarking competitivo foi destinado a investigar o seguinte questionamento: quais são os aplicativos voltados à terapia farmacológica e aos cuidados dos pacientes transplantados renais?

A busca e a seleção pelos aplicativos foram realizadas de fevereiro a março de 2020, nas duas principais lojas virtuais de aplicativos - Android (Google play) ${ }^{1}$ e iOS (App Store) ${ }^{2}$. O processo de busca se deu da seguinte forma: Google play, os aplicativos foram identificados na categoria "Medicina" e "Saúde fitness", utilizando os descritores em português e inglês: "Transplante renal/ Kidney transplant" e "Transplante renal medicações/ Kidney transplant medications". Já na Apple store foram identificados os aplicativos Apple mais populares, gratuitos, dispostos nas categorias "Medicina" e "Saúde fitness". Foram categorizados os aplicativos instaláveis em iPhone, iPad ou outro aparelho que suporte alguma versão do sistema operacional iOS e utilizados os mesmos descritores em ambos os idiomas.

\footnotetext{
${ }^{1}$ Link da loja virtual Google Play. https://play.google.com/store

${ }^{2}$ Link da loja virtual App Store: https://www.apple.com/br/ios/ app-store/
} 
Foram adotados como critérios de inclusão: os aplicativos disponíveis gratuitamente; disponíveis nas lojas virtuais Google play e App Store, adaptados para dispositivos Tablets ou Smartphones, em idioma português, inglês ou espanhol. Foram excluídos os aplicativos com restrição no Brasil, duplicados, aplicativos não direcionados à terapia medicamentosa e aos cuidados aos pacientes transplantados renais e os quais apresentaram problemas funcionais e/ou técnicos.

$\mathrm{Na}$ segunda etapa, os aplicativos em análise foram tabulados e avaliados quanto à plataforma virtual, idioma e usabilidade. Para checar a usabilidade dos aplicativos, os mesmos foram baixados, instalados e verificados sobre sua usabilidade e pertinência com o assunto proposto pelo desenvolvedor, destacando a necessidade das características serem compatíveis com o público alvo. Posteriormente, os aplicativos selecionados foram avaliados pelos pesquisadores, por meio do instrumento/questionário System Usability Scale (SUS), criado por John Brooke em 1986 e validado no Brasil por Tenório et al. ${ }^{8,9}$.

Os aplicativos analisados foram instalados nos hardwares compatíveis com as respectivas lojas virtuais. Dessa forma, para o sistema Android foi utilizado o aparelho Samsung Galaxy A5 4G Duos SM-A500M/DS, e para o sistema iOS o Apple iPhone Xs Max (Unlocked) 64GB - Gold MT5X2LL/A.

O escore SUS é uma ferramenta que possui capacidade de avaliar websites, serviços, softwares e aplicações. É composta por dez itens com cinco opções de resposta (discordo totalmente, discordo, neutro, concordo e concordo totalmente), variando as respostas de um (discordo totalmente) a cinco (concordo totalmente), possuindo facilidade e praticidade de resposta. Esta ferramenta permite avaliar de forma ampla a diversidade de produtos e serviços.

Para o cálculo do escores SUS, inicialmente soma-se a resposta de cada item (escala de 1 a 5). Para as questões ímpares (1,3,5,7 e 9), o escore obtido é a resposta fornecida individualmente menos um. Para as questões pares (2,4,6,8 e 10), subtraia-se a resposta de cinco. Somam-se todos os valores das dez perguntas e o resultado obtido é multiplicado por 2.5. A pontuação final será o índice de satisfação do utilizador, um número que varia de 0 a 100 e pode ser enquadrado em uma das seguintes categorias: 20,5 (pior imaginável); 21 a 38,5 (pobre); 39 a 52,5 (mediano); 53 a 73,5 (bom); 74 a 85,5 (excelente); e 86 a 100 (melhor imaginável) $)^{8,10}$.

$\mathrm{Na}$ terceira etapa, utilizou-se a metodologia SCAMPER, aplicada conjuntamente ao benchmarking. A metodologia SCAMPER é uma técnica utilizada em processos ou produtos já existentes para proporcionar novas ideias por meio de um checklist proposto. Além disso, 
busca solucionar problemas existentes nos produtos já desenvolvidos que não possuíram sucesso significativo ${ }^{11}$.

O estudo foi submetido à apreciação do comitê de ética e pesquisa (CEP) do Hospital Geral de Fortaleza, o qual foi avaliado sua viabilidade de sua realização e sendo aprovado sob o número de parecer 3.934.154.

\section{Resultados}

\subsection{Estado da arte e Benchmarking}

A investigação dos aplicativos se deu por meio dos descritores em português e inglês e foram identificados os aplicativos inclusos nas categorias "medicina" e "saúde fitness". O maior número de aplicativos foi encontrado na plataforma Google Play, com 919 aplicativos disponíveis para download. $\mathrm{Na}$ Apple Store foram encontrados 24 aplicativos. Para melhor organização dos resultados, foi utilizado o protocolo PRISMA (Figura 1) ${ }^{12}$ (Anexos).

Inicialmente foram encontrados 943 aplicativos como resultado da busca realizada com auxílio dos descritores. Destes aplicativos, 219 foram excluídos por serem duplicados, o que resultou em 724 aplicativos para próxima avaliação, tendo sido analisados quanto ao título, descrição do desenvolvedor e temática. Outros 617 aplicativos foram excluídos por não atenderem ao tema proposto. Dos 107 aplicativos elegíveis, foram excluídos 100 por não responderem aos critérios de idioma e gratuidade. Por fim, foram realizados os downloads e instalados para avaliação integral, atendendo aos critérios de inclusão e exclusão, sete aplicativos que fizeram parte da seleção final.

$\mathrm{Na}$ seleção final, os aplicativos foram avaliados criteriosamente, por meio do benchmarking competitivo, sendo verificadas as características e funcionabilidade de cada aplicativo incluso dentro da revisão. Dos sete aplicativos selecionados, quatro foram na plataforma Google Play, sendo três em língua inglesa e um em espanhol, e três aplicativos na plataforma Apple Store, todos na língua inglesa (Quadro 1) (Anexos).

A partir dos resultados obtidos, pôde-se verificar a predominância da língua inglesa em $86 \%$ dos aplicativos, sendo que apenas um estava na língua espanhola, restringindo o acesso dos pacientes, fazendo-se necessário o desenvolvimento de aplicativos em outros idiomas. 0 estudo de Barra et al. ${ }^{13}$, também evidenciou um número deficiente de aplicativos na língua portuguesa, corroborando para a necessidade de adaptação ou ampliação da disponibilidade para outros idiomas. 
O APP Kidney Transplant Medications (Eng), disponível no Google Play, contém recursos interativos, didáticos e ilustrativos, enfatizando o processo de rejeição por meio de figuras, fazendo analogias com os possíveis eventos adversos dos medicamentos, utilizando textos curtos e alertas de cuidado para o paciente. Dispõe adicionalmente de informações sobre terapia associada ao transplante.

O APP Kidney Transplant Education, desenvolvido pelo Centro de Transplante da Universidade de Michigan, destaca a função do farmacêutico dentro da equipe no acompanhamento terapêutico pré e pós-transplante. O APP fornece orientações nutricionais, recomendações de como enfrentar descargas emocionais, dificuldades financeiras, incluindo direcionamentos sobre os cuidados pós-transplante. Apresenta uma aba sobre medicamentos no transplante e um link disponível sobre o serviço de farmácia em Michigan. Possui um espaço descrevendo toda a equipe, os principais contatos em caso de urgência e/ou dúvidas e o portal do paciente.

O Our Journey with a Kidney Transplant destina-se às famílias e cuidadores de pacientes pediátricos com transplante renal. Dispõe de conteúdo sobre prevenção da rejeição aguda, pré-transplante, exames e suas funções e o preparo para os procedimentos. Sobre a terapia farmacológica, identifica os medicamentos, seus efeitos adversos e como evitá-los. Soma-se a isto informações sobre a dispensação, armazenamento e administração dos imunossupressores. O APP apresenta limitações referentes ao uso de dados móveis, linguagem complexa e textos em PDF. No entanto, utiliza-se de cores atrativas e ferramentas de caixa de anotações.

O Transplant Hero contempla a estratégia de adesão à imunossupressão. O APP dispõe de recursos interativos e seu foco principal é o alarme no horário correto das medicações, sendo capaz de ajustar toda terapia medicamentosa do paciente. Apresenta recursos de interação com o paciente, por meio de recompensas didáticas. Logo, o paciente sente-se interessado em seguir plenamente os horários posológicos.

O Piedmont Transplant Institute, considerado um centro transplantador de referência, desenvolveu o Piedmont Kidney Pancreas, que possui recomendações sobre processos de doação e compatibilidade para profissionais e pacientes. O APP também dispõe de um quiz com perguntas frequentes sobre a doação intervivos, a qual é uma realidade nos Estados Unidos, com cerca de 1/3 das doações. Além disso, estabelece orientações de alta após o transplante, orientações sobre os imunossupressores, atividades sexuais, nutricionais e protocolos terapêuticos. Contudo, o APP apresentou falha nos vídeos disponíveis, além de informações complexas aos pacientes. 
O PatientMpower é um APP informativo e dinâmico, que faz o acompanhamento diário de exames laboratoriais, sintomas e administração de medicamentos, além do monitoramento dos níveis séricos dos imunossupressores. Sistematiza todos os dados e fornece o compilado das informações por meio de gráficos e relatórios diários, semanais, mensais e anuais. Por outro lado, o APP requer elevado número de informações, necessitando que o paciente as preencha, fazendo-se necessário fornecer instruções aos pacientes que irão utilizá-lo.

O APP Transplant Coach - Illuminate, direcionado para pacientes pós transplante, tem como estratégia central a adesão à terapia medicamentosa. A ferramenta apresenta métodos para diferenciação dos medicamentos, sendo capaz de personalizar todos os fármacos e inserir a terapia na rotina, além de fornecer ao paciente orientações de cada medicamento adicionado. Soma-se a isto recursos para adicionar os prescritores, farmácias e contatos emergenciais.

A acessibilidade dos aplicativos é limitada para muitos usuários quando necessita dispor do uso de internet e/ou dados móveis, sendo uma realidade nos aplicativos que compõem esse estudo, o que dificulta o acesso ou até mesmo priva o paciente de desfrutar de algumas funções. 0 estudo de Watanabe et al. ${ }^{14}$, ressalta que aplicativos direcionados para terapia farmacológica que fazem uso da internet constituem um obstáculo para diversos pacientes.

\subsection{Avaliação da usabilidade.}

Os APP baixados foram analisados com o instrumento System Usability Scale (SUS) para avaliar a usabilidade. Segundo Tenório et al. ${ }^{9}$, este instrumento consiste em um questionário capaz de avaliar produtos, interfaces, softwares, websites e aplicações, além de critérios de efetividade, eficiência e satisfação, onde são identificadas as inconsistências e benefícios através do uso prévio dos aplicativos selecionados. 0 quadro 2 (Anexos) detalha a avaliação de cada aplicativo submetido à análise.

Os aplicativos demonstraram níveis de usabilidade variáveis e apenas três aplicativos (42,9\%), atingiram o escore de corte de 68 pontos, dito como satisfatório. Apesar de alguns aplicativos terem sido classificados como de excelente usabilidade, requerem adequações e ajustes para integralmente atender às necessidades dos usuários e compreender às dificuldades que possam vir a surgir após o uso dos aplicativos móveis. Consequentemente, o processo de usabilidade deve ser contínuo e dinâmico para alcançar e minimizar as dificuldades de uso dos mesmos ${ }^{15}$. 
Visto que quatro aplicativos $(57,1 \%)$ não alcançaram a média, pôde-se inferir que os aplicativos apresentam complicações de usabilidade, característicos de sistemas com instabilidade e difícil manejo. Os resultados do estudo foram semelhantes aos de Caivano, Ferreira, Domene ${ }^{16}$, em que $41,24 \%$ dos usuários sugeriram melhorias na usabilidade. Em contrapartida, o APP Guia Alimentar Digital (GAD) do estudo de Caivano dispõe de boa usabilidade, além de ser uma ferramenta intuitiva e autoexplicativa.

Através dos resultados encontrados observou-se um número restrito de APPs no idioma português e espanhol, em oposição à multiplicidade de APPs no idioma inglês. Além disto, evidenciou-se um elevado número de APPs voltados apenas para profissionais de saúde e conteúdos profissionais, como guidelines e livros. Tibes et al. ${ }^{17}$ realizaram uma revisão integrativa, na qual avaliaram diversos aplicativos utilizados na saúde. Demonstraram uma diversidade de estudos com foco multiprofissional, com objetivo didático e teórico direcionados apenas para os profissionais, encontrado apenas três estudos com foco no paciente, todos destinados ao uso direto para o gerenciamento de doenças, desempenhando suporte remoto.

Os APPs carecem de conteúdos e linguagem adequados para pacientes e usuários com baixa escolaridade, além de elementos básicos de autocuidado embasados na prática clínica e baseados em evidências. Estes fatores podem dificultar a usabilidade e podem repercutir negativamente na adesão do paciente ao aplicativo. Portanto, é necessário conteúdo direcionado ao público-alvo, destacando-se a importância de conhecer as deficiências e projetar tecnologias direcionadas e adequadas aos usuários ${ }^{18}$.

Os APPs estão bastantes difundidos no âmbito assistencial de saúde. Destacam-se no autogerenciamento de doenças crônicas direcionados aos pacientes, familiares e cuidadores. Estudos mostram que essas tecnologias móveis são eficazes e servem de suporte para o tratamento de patologias, além de diminuírem a não conformidade da terapia a ser seguida ${ }^{19}$.

Com o elevado número de APPs disponíveis em lojas virtuais direcionados aos pacientes com doenças crônicas, faz-se necessário avaliar a usabilidade pelo público-alvo. Nesse contexto, a usabilidade dos APP é insegura, não sendo representativa para pacientes com classe econômica baixa, escolaridade inferior e para idade avançada ${ }^{19}$.

Determinar a usabilidade de APPs móveis é complexo. Estudos mostram que é necessário criar habilidades para analisar a percepção e verificar a funcionabilidade e atividades operacionais. Nas informações disponíveis nos APPs, prevalecem aquelas voltadas à equipe médica e equipes multiprofissionais, com orientações limitadas aos 
pacientes sobre autocuidado e seguimento correto da terapia medicamentosa e não farmacológica ${ }^{20}$.

A tecnologia móvel demonstra ser aceitável e compreensível aos pacientes que a utilizam para o gerenciamento de doenças e para o tratamento farmacológico, sendo os APP uma forma de melhorar a eficiência no cuidado da saúde. Entretanto, os desenvolvedores e pesquisadores precisam fornecer conteúdos confiáveis e seguros para os pacientes ${ }^{19,20}$.

A busca resultou em um vasto número de aplicativos, reforçando a importância da usabilidade frente a variedade dos mesmos, sendo imprescindível que se tenha maior direcionamento de conteúdo e atividades funcionais aos pacientes, além de ter capacidade para transmitir informações e aumentar a satisfação e confiança dos usuários. Atualmente a usabilidade estabelece melhor interface entre os usuários e o ambiente tecnológico ${ }^{20}$.

\subsection{Técnica SCAMPER}

A fase final do estudo caracterizou-se pela certificação e melhorias propostas aos aplicativos em estudo, utilizando metodologias distintas com intuito único e efetivo, sendo capaz de implementar ideias inovadoras e elaborar ferramentas com capacidade singular aos pacientes transplantados. A técnica SCAMPER possibilitou a transparência entre as possíveis transformações e as modificações favoráveis e oportunas para as tecnologias móveis, de acordo com o Quadro 3 (Anexos).

A utilização da técnica SCAMPER encontra justificativa neste contexto por promover a motivação e a produção de ideias, com a finalidade de provocar impacto de modificações, além de ser usada para prosperar a criatividade e auxiliar na tomada rápida de decisões e na viabilidade de ideias inovadoras ${ }^{21}$.

Diante das possíveis modificações foram elencados tópicos que sejam capazes de desenvolver melhorias competitivas aos aplicativos já existentes, resultando em um aplicativo com funcionabilidade e originalidade ímpar. Por meio da análise, os APP demonstraram conteúdos complexos e textos de cunho científico, permeando entre os usuários dificuldades no entendimento, sendo proposto a substituição para conteúdos didáticos e dinâmicos, apresentado por figuras e/ou desenhos ilustrativos, além de facilitar o acesso aos aplicativos por meio de cadastros simples e objetivos.

Alguns dos APP geram relatórios de acordo com as informações fornecidas pelos usuários como: características sociodemográficas, exames e resultados. Faz-se necessário combinar um sistema interativo de envio dos relatórios para equipe multiprofissional do 
transplante ou mesmo os profissionais terem acesso por outros meios, além de desenvolver ferramentas para administração dos dados. Soma-se a isto, fornecer a interação de conteúdo por meio de ferramentas audiovisuais. Siddique et al. ${ }^{22}$ obtiveram resultados semelhantes, evidenciando que a maioria dos aplicativos receberam pontuação baixa devido à falta de recursos interativos e personalização dos dados.

A ausência de APP em idioma português acarreta déficit de informações para determinados grupos de pacientes, fazendo-se necessário adaptar aos APP já existentes ou em desenvolvimento, a função de escolha do idioma, estabelecendo apreciação de grupos de várias nações. Vídeos estão presentes em várias plataformas. A adaptação dos vídeos se dá em temas pertinentes aos pacientes com transplante, com foco na adesão e benefícios dos imunossupressores, fazendo com que seja comtemplado por pacientes de idades distintas e para diferentes modalidades de transplante.

A visibilidade e a existência de determinados aparatos tecnológicos em saúde necessitam ser difundido por intermédio da divulgação em eventos científicos, publicações em revistas de impacto considerável e em mídias sociais. É fundamental a disponibilização e a divulgação de tecnologias em saúde para os pacientes terem acesso as tecnologias desenvolvidas em prol de seus benefícios. 0 estudo de Formagini et al. ${ }^{23}$ discute sobre as tecnologias móveis serem promissoras aos pacientes por facilitarem as intervenções e a educação em saúde, além de possuírem grande alcance remoto, facilitando o aconselhamento de vários usuários simultaneamente.

Grande parte das instabilidades existentes nas plataformas apresenta-se por meio de downloads e uso de dados móveis, como por exemplo, uso de internet. Apesar da evolução e do crescente uso da Internet, isto acarreta limitações e dificulta a usabilidade. Ter acesso ao conteúdo e a ferramenta tecnológica apenas com uso da internet restringe o usuário, além da possibilidade de intercorrências e oscilações no sistema, fazendo-se necessário simplificar e difundir o acesso com maior facilidade aos usuários. Em um estudo de revisão de aplicativos para DRC e transplante renal, os APP receberam boa pontuação para usabilidade, entretanto apresentaram inconsistência devido à falta de recursos de personalização, além de hiperlinks com falhas técnicas e bugs ${ }^{22}$.

Diante das tecnologias e plataformas móveis já disponíveis, a técnica SCAMPER surge da teoria "Toda ideia é nascida de outras ideias existentes", sendo essa filosofia repassada para ciência tecnológica com a finalidade de buscar melhorias e implementar ideias que ainda não foram desenvolvidas na atualidade ${ }^{24}$. 
O checklistproposto pela técnica SCAMPER é capaz de melhorar o potencial criativo. Soma-se a isto a necessidade de aperfeiçoar habilidades, técnicas e ferramentas cognitivas, com propósito de desenvolver e aprimorar objetos e dispositivos alternativos a partir de uma ideia já existente ${ }^{25}$.

\section{Conclusão}

Neste estudo foram apresentados os aplicativos disponíveis direcionados para pacientes transplantados renais. A maioria dos aplicativos selecionados estava indisponível na língua portuguesa, dificultando o acesso aos pacientes. A usabilidade apresentou-se satisfatória, entretanto, faz-se imprescindível adaptação, planejamento e desenvolvimento de novas tecnologias compatíveis e efetivas, em concordância com as necessidades e demandas dos usuários.

A técnica SCAMPER proporcionou novas ideias e melhorias para os aplicativos em saúde. É indispensável o desenvolvimento de novas ferramentas tecnológicas para o controle farmacológico e adesão medicamentosa para os pacientes, proporcionando o autogerenciamento de doenças crônicas e o conhecimento sobre sua própria saúde.

A revisão sistemática nas lojas virtuais, avaliação de usabilidade dos APP através do questionário SUS e a técnica SCAMPER foram estratégias capazes de evidenciar processos metodológicos e tecnológicos ainda não desenvolvidos nos APP já disponíveis, e conceber ideias inovadoras ao protótipo destinado aos pacientes transplantados, atestando melhor eficiência ao produto tecnológico; foram ainda capazes de fornecer informações seguras e desenvolver estratégias para orientação e adesão medicamentosa aos pacientes transplantados renais.

\section{Referências}

1. Pinto LF, Rocha CMF. Inovações na atenção primária em saúde: $O$ uso de ferramentas de tecnologia de comunicação e informação para apoio à gestão local. Cienc e Saude Coletiva. 2016;21(5):1433-48.

2. Galván P, Rivas R, Ortellado J, Portillo J, Mazzoleni JHE. Rev. salud publica Parag. Vol. 10 $N^{\circ} 1$ Ene - Jun 2020 Galván P, et al.. "Aplicación de tecnologías disruptivas en telemedicina." 2020;10(1):52-8.

3. Velasco HF, Cabral CZ, Pinheiro PP, Azambuja RDCS, Vitola LS, Costa MR Da, et al. Use of digital media for the education of health professionals in the treatment of childhood asthma. J Pediatr (Rio J) [Internet]. 2015;91(2):183-8. Available from:

http://dx.doi.org/10.1016/j.jped.2014.07.007

4. Vieira LB, Ramos CDÁ, Castello MDB, Do Nascimento LC. Development of an electronic device to organize medications and promote treatment adherence. Rev Panam Salud 
Publica/Pan Am J Public Heal. 2016;39(4):208-12.

5. Gong K, Yan Y-L, Li Y, Du J, Wang J, Han Y, et al. Mobile health applications for the management of primary hypertension. Medicine (Baltimore). 2020;99(16):e19715.

6. Oliveira GG. Proposição de um sistema de informação de benchmarking para empresas desenvolvedoras de produtos. pgdesign. 2017;1:1-322.

7. Ferreira RC, Lanne G, Luna M, Bezerra AG. Benchmarking na identificação de aplicativos móveis internacionais sobre a violência na escola. 2016;4:138-43.

8. John Brooke. SUS - A quick and dirty usability scale. Usability Eval Ind. 1996;189-94.

9. Tenório JM, Cohrs FM, Sdepanian VL, Pisa IT, Marin HDF. Desenvolvimento e Avaliação de um Protocolo Eletrônico para Atendimento e Monitoramento do Paciente com Doença Celíaca. Rev Informática Teórica e Apl. 2011;17(2):210.

10. Marimon BR, Margarida Rockenbach TL. Avaliação de Ambiente Virtual de Aprendizagem com o uso do SUS - System Usability Scale. Renote. 2014;11(3).

11. Taifa IWR, Desai DA, Bulsara NM. The development of an ergonomically designed product through an integrated product team approach. Int J Occup Saf Ergon [Internet]. 2019;0(0):1-32. Available from: https://doi.org/10.1080/10803548.2018.1557398

12. Moher $D$, Liberati A, Tetzlaff J AD. Principais itens para relatar Revisões sistemáticas e Meta-análises: A recomendação PRISMA. Epidemiol e Serviços Saúde. 2015;24(2):335-42. 13. Barra DCC, Paim SMS, dal Sasso GTM, Colla GW. Métodos para desenvolvimento de aplicativos móveis em saúde: Revisão integrativa da literatura. Texto e Context Enferm. 2017;26(4):1-12.

14. Watanabe N, Horikoshi M, Yamada M, Shimodera S, Akechi T, Miki K, et al. Adding smartphone-based cognitive-behavior therapy to pharmacotherapy for major depression (FLATT project): Study protocol for a randomized controlled trial. Trials. 2015;16(1). 15. Suzart F, Santana EB, Santos É, Silva J, Carvalho M, Luís F, et al. Uso De Apps Para a Promoção Dos Cuidados À Saúde. An do Semin Tecnol Apl a Educ e Saúde. 2017;0(0). 16. Caivano S, Ferreira BJ, Domene SMÁ. Avaliação da usabilidade do Guia Alimentar Digital móvel segundo a percepção dos usuários. Cienc e Saude Coletiva.

2014;19(5):1437-46.

17. Tibes CM dos S, Dias JD, Zem-Mascarenhas SH. Mobile applications developed for the health sector in Brazil: an integrative literature review. REME Rev Min Enferm.

2014;18(2):471-8.

18. Nívea TATN, Balduíno DAY, Melo CC, Leite DE, Cardoso S, Matias E, et al. Tendências De Estudos Sobre Aplicativos Móveis Para Saúde: Revisão Integrativa. XV Congr Bras Informática em Saude [Internet]. 2016;499-508. Available from: www.jhisbis.saude.ws/ojs-jhi/index.php/jhi-sbis

19. Sarkar U, Gourley GI, Lyles CR, Tieu L, Clarity C, Newmark L, et al. Usability of Commercially Available Mobile Applications for Diverse Patients. J Gen Intern Med [Internet]. 2016;31(12):1417-26. Available from: http://dx.doi.org/10.1007/s11606-0163771-6

20. Yasini M, Marchand G. Mobile Health Applications, in the Absence of an Authentic Regulation, Does the Usability Score Correlate with a Better Medical Reliability? Stud Health Technol Inform. 2015;216:127-31.

21. Serrat O, Serrat O. The SCAMPER Technique. In: Knowledge Solutions. Springer Singapore; 2017. p. 311-4.

22. Siddique AB, Krebs M, Alvarez S, Greenspan I, Patel A, Kinsolving J, et al. Mobile apps for the care management of chronic kidney and end-stage renal diseases: Systematic search. J Med Internet Res. 2019;21(9):1-9.

23. Formagini TDB, Ervilha RR, Machado NM, Andrade BABB de, Gomide HP, Ronzani TM. Revisão dos aplicativos de smartphones para cessação do tabagismo disponíveis em 
língua portuguesa. Cad Saude Publica. 2017;33(2):1-10.

24. Cahyati $H$, Muin A, Musyrifah E. Efektivitas Teknik SCAMPER dalam Mengembangkan Kemampuan Berpikir Kreatif Matematis Siswa. J Medives J Math Educ IKIP Veteran Semarang. 2018;2(2):173.

25. Özyaprak M. The effectiveness of SCAMPER technique on creative thinking skills. $J$ Educ Gift Young Sci. 2016;4(1):31-40. 


\section{Anexos}

Figura 1: Fluxograma de seleção dos aplicativos inclusos na revisão, de acordo com o protocolo PRISMA.

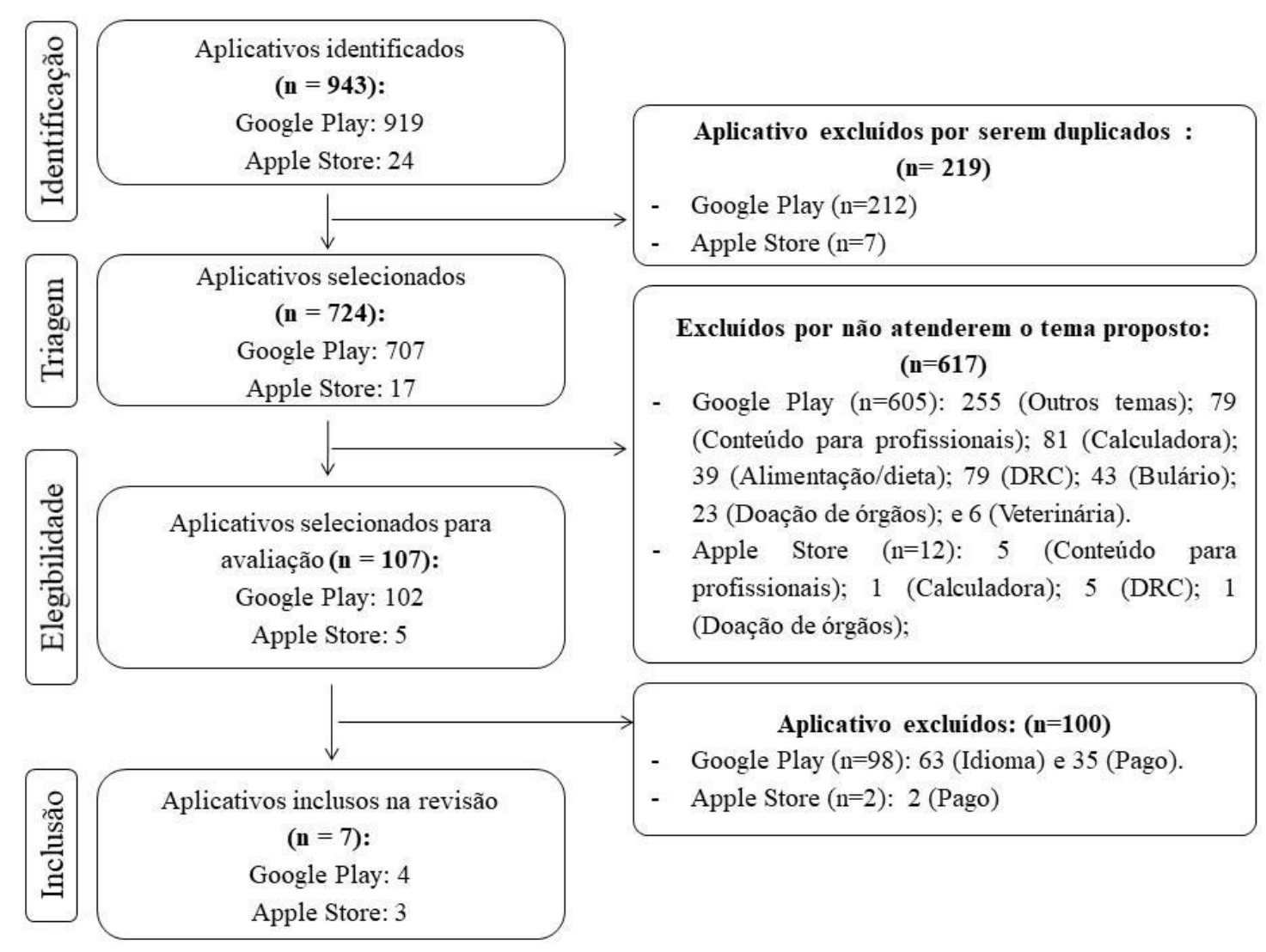

Fonte: os autores.

Quadro 1: Caracterização dos aplicativos selecionados.

\begin{tabular}{|c|c|c|c|}
\hline CÓDIG0 & APLICATIVO & PLATAFORMA & IDIOMA \\
\hline AP1 & Kidney Transplant Medications (Eng) & Google play & Inglês \\
\hline AP2 & Kidney Transplant Education & Google play & Inglês \\
\hline AP3 & Our Journey with a Kidney Transplant & Google play & Espanhol \\
\hline AP4 & Transplant Hero & Google play & Inglês \\
\hline AP5 & Piedmont Kidney Pancreas & Apple store & Inglês \\
\hline AP6 & PatientMpower & Apple store & Inglês \\
\hline AP7 & Transplant Coach - Illuminate & Apple store & Inglês \\
\hline
\end{tabular}

Fonte: os autores. 
15

Quadro 2: Avaliação de usabilidade dos aplicativos selecionados na busca sistemática, através do instrumento SUS.

\begin{tabular}{|c|c|c|c|}
\hline CÓDIGO (APP) & SISTEMA OPERACIONAL & SUS & AVALIAÇÃO \\
\hline AP1 & Android (Google Play) & 50 & Mediano \\
\hline AP2 & Android (Google Play) & 75 & Excelente \\
\hline AP3 & Android (Google Play) & 65 & Bom \\
\hline AP4 & Android (Google Play) & 80 & Excelente \\
\hline AP5 & iOS (Apple Store) & 52,5 & Mediano \\
\hline AP6 & iOS (Apple Store) & 60 & Bom \\
\hline AP7 iOS (Apple Store) & 75 & Excelente \\
\hline \multicolumn{4}{|c|}{ A média do System Usability Score (SUS) é 68 pontos. } \\
\hline
\end{tabular}

Fonte: os autores.

Quadro 3: Sugestões e modificações por meio do checklist SCAMPER.

\begin{tabular}{|c|c|}
\hline TRANSFORMAÇÕES & MODIFICAÇÕES \\
\hline Substituir & $\begin{array}{l}\text { - Conteúdo e linguagem complexa; } \\
\text { - Imagens reais por figuras ilustrativas e/ou desenhos; } \\
\text { - Cadastros longos e difíceis por cadastros simples; }\end{array}$ \\
\hline Combinar & $\begin{array}{l}\text { - Cadastros e senhas para acessar os dados em desktop; } \\
\text { - Envio de informações e relatórios para os profissionais } \\
\text { de saúde; } \\
\text { - Recursos visuais e auditivos; }\end{array}$ \\
\hline Adaptar & $\begin{array}{l}\text { - Os APP em vários idiomas (disponibilizar opções de } \\
\text { vários idiomas); } \\
\text { - Vídeos explicativos sobre o risco de não adesão a } \\
\text { imunossupressão; } \\
\text { - Conteúdos para diversos públicos de pacientes e idades; } \\
\text { - Para outros tipos de transplantes; }\end{array}$ \\
\hline $\begin{array}{c}\text { Modificar/Magnificar/Mi } \\
\text { nificar }\end{array}$ & $\begin{array}{l}\text { Conteúdo direcionado para profissionais de saúde, } \\
\text { disponibilizando conteúdos com o foco no usuário; } \\
\text { - Disponibilizar para outros sistemas operacionais; }\end{array}$ \\
\hline Por em outros usos & $\begin{array}{l}\text { - Disponibilizar o APP em publicações, eventos científicos } \\
\text { e mídias sociais; } \\
\text { - Disponibilizar para outros tipos de pacientes } \\
\text { transplantados; }\end{array}$ \\
\hline Eliminar & $\begin{array}{l}\text { - Conexão de dados para acessar o aplicativo; } \\
\text { - Apenas para uso profissional, ou seja, ampliar a } \\
\text { disponibilização; }\end{array}$ \\
\hline Rearranjar/Reverter & $\begin{array}{l}\text { - Gráficos, tabelas e relatórios dos dados gerados; } \\
\text { - Após os resultados e sugestões dos especialistas; }\end{array}$ \\
\hline
\end{tabular}

Fonte: os autores. 